Research Paper

\title{
The Expression of Ubiquitous Mitochondrial Creatine Kinase Is Downregulated as Prostate Cancer Progres- sion
}

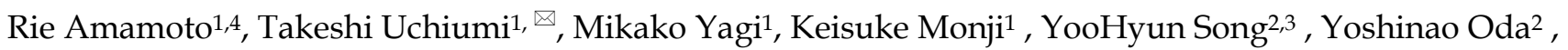 \\ Masaki Shiota $^{3}$, Akira Yokomizo ${ }^{3}$, Seiji Naito ${ }^{3}$, Dongchon Kang ${ }^{1}$ \\ 1. Department of Clinical Chemistry and Laboratory Medicine \\ 2. Department of Anatomic Pathology \\ 3. Department of Urology, Kyushu University, Fukuoka, Japan \\ 4. Department of Nutritional Sciences, Seinan Jo Gakuin University, Kitakyushu, Japan \\ $\triangle$ Corresponding author: Department of Clinical Chemistry and Laboratory Medicine, Graduate School of Medical Sciences, Kyushu University, 3-1-1 Mai- \\ dashi, Fukuoka 812-8582, Japan. Tel: (+81) 92642 5752; Fax: (+81) 92642 5772; E-mail: uchiumi@cclm.med.kyushu-u.ac.jp \\ (1) Ivyspring International Publisher. Reproduction is permitted for personal, noncommercial use, provided that the article is in whole, unmodified, and properly cited. See \\ http://ivyspring.com/terms for terms and conditions.
}

Received: 2015.07.10; Accepted: 2015.08.31; Published: 2016.01.01

\begin{abstract}
Background: Mitochondria play crucial roles in cell signaling events, interorganellar communication, aging, cell proliferation and apoptosis, and mitochondrial impairment has been shown to accelerate or modulate cancer progression. Ubiquitous mitochondrial creatine kinase (uMtCK) is predominantly localized in the intermembrane space of mitochondria and catalyzes the reversible exchange of high-energy phosphate between adenosine tri-phosphate (ATP) and phosphocreatine. However, little is known about its expression and function in human prostate cancer progression.

Method: We investigated the expression of uMtCK in 148 prostate carcinoma tissues and matched normal tissue by immunohistochemistry. The expression and localization of uMtCK and hexokinase II, a marker of glycolysis, were examined in prostate carcinoma cell lines using western blot and immunofluorescence.

Results: MtCK expression was significantly lower in high Gleason grade carcinoma compared with normal prostate or low grade carcinoma. Western blot further revealed that uMtCK was highly expressed in LNCaP and $22 \mathrm{Rv} 1$ cell lines, as well as in the normal prostate cell line RWPE-1. However, uMtCK expression was almost absent in PC3 and DU145 cell lines, in correlation with absent or mutant $\mathrm{p} 53$ expression, respectively. In contrast, hexokinase II was overexpressed in PC3 cells. Moreover, in the low uMtCK expressing cell lines, glycolytic ATP production was increased, whereas mitochondrial ATP production was decreased.

Conclusions: These data suggest that uMtCK is downregulated as prostate cancer progresses in correlation with a metabolic switch in ATP usage.
\end{abstract}

Key words: mtCK, ATP, mitochondria

\section{Introduction}

Prostate cancer is the most common noncutaneous cancer and the second leading cause of male cancer-related mortality in developed countries[1]. Nutritional and lifestyle factors influence the risk of prostate cancer[2, 3]. Prostate carcinogenesis is a complex process as reflected in the heterogeneity of its clinical and pathological diagnosis, and in the long latency in the development of life-threatening prostate cancer[4]. A better understanding of the mechanisms underlying prostate tumor growth and the identification of novel molecular markers implicated in its progression are thus needed to determine new therapeutic approaches.

The Warburg effect represents a prominent 
metabolic characteristic of malignant cells. A phenomenon first described by Otto Warburg, the Warburg effect is a metabolic phenotype characterized by a shift from oxidative phosphorylation (OXPHOS) to aerobic glycolysis as the main source of ATP production[5]. Although mitochondrial respiration is decreased in cancer cells, Warburg speculated that it is essential for cancer cell survival.

Creatine kinase (CK) isoenzymes catalyze the reversible transfer of phosphate groups from phosphocreatine to ADP, to yield ATP and creatine. Different types of CKs are found in the cytosol and mitochondria of cells[6]. There are two forms of mitochondrial CKs (MtCK), sarcomeric MtCK (sMtCK) and ubiquitous MtCK (uMtCK), that are encoded by two genes and are expressed in a tissue-specific manner[7]. In vertebrates, sMtCK is found in striated muscle. In contrast, uMtCK is co-expressed with cytosolic brain-type subunits in many cells and tissues with high energy demand, such as the brain, placenta, kidney, testis, sperm, and endothelial cells[8]. Indeed, uMtCK is a central controller of cellular energy homeostasis[9].

MtCKs are primary targets of oxidative-induced molecular damage, and their dysfunction has been reported in ischemia, cardiomyopathy, and neurodegenerative disorders $[9,10]$. For example, upregulation of MtCK has been shown in creatine-depleted muscles and in patients with mitochondrial cytopathies, in which it may act in a compensatory role to overcome functional impairment of the energy state control. Notably, overexpression of MtCK in tumors has also been reported[11, 12].

In the glycolytic pathway, hexokinase (HK) catalyzes the essentially irreversible first step, in which glucose is phosphorylated to glucose-6-phosphate (G-6-P) via phosphate transfer from ATP[13]. HKII, the predominant isoform overexpressed in malignant tumors, is strategically located on the outer mitochondrial membrane protein voltage-dependent anion channel (VDAC)[14].

Because uMtCK is a key molecule for OXPHOS and apoptosis in mitochondria, it might be involved in cancer progression. Indeed, the expression of uMtCK is significantly increased in some human cancer tissues. However, little is known about the role of $\mathrm{uMtCK}$ in prostate cancer progression and mitochondrial function. In this study, we investigated the expression of uMtCK in patient-derived prostate cancer tissues by immunohistochemistry and found that its expression showed a strong negative correlation with clinicopathological parameters. We also found that uMtCK expression was significantly decreased in high Gleason grade cells compared with low Gleason grade prostate cancer cell lines or normal prostate cells. Furthermore, in high Gleason grade prostate cancer cells, whereas mitochondrial expression of uMtCK was decreased, HKII expression increased, indicative of a change from OXPHOS to glycolysis. We thus propose that uMtCK may be a novel progressive marker of prostate cancer.

\section{Materials and Methods}

\section{Patients, tissues and clinicopathological data}

Tumor biopsies were collected from 148 patients (mean age: 65.6 years, range: $47-78$ years) who received radical prostatectomy for clinically localized prostate cancer at the Kyushu University Hospital, Fukuoka, Japan, between 1997 and 2006. All patients were diagnosed according to the outcomes of prostate specific antigen (PSA) concentration, digital rectal examination, and prostate needle biopsy. No patient received chemotherapy or hormonal therapy before surgery. Detailed clinicopathological features of the patients are summarized in Supplemental Table S1. All prostatectomy specimens were reviewed to establish stage and grade of the respective prostate cancers, as previously described[15]. The clinical follow-up data were available for 128 patients and included monitoring of serum PSA concentration. A PSA level greater than $0.4 \mathrm{ng} / \mathrm{ml}$ was defined as PSA recurrence, and PSA recurrence was found in 20\% (26/128) of patients. The median follow-up time of patients still relapse-free at the end of analysis was 49.5 months[16]. This study was conducted in accordance with the principles embodied in the Declaration of Helsinki, the Ethical Guidelines for Human Genome/Gene Research enacted by the Japanese Government, and the institutional review board approval.

\section{Antibodies}

Anti- $\beta$-actin antibody was purchased from Sigma-Aldrich (St. Louis, MO, USA). Anti-uMtCK and anti-sMtCK antibodies were a gift from Shino Test Corporation (Tokyo, Japan). Two antibodies against p53 (DO-1 and FL393) were purchased from Santa Cruz (Dallas, TX, USA). Polyclonal antibodies against mouse p32 and VDAC were generated in our laboratory as described previously[17]. Antibodies against COXI, COXII, NDUFA9 (complex I), SDHA (complex II), UQCRFS1 (complex III), and complex V were purchased from Invitrogen (Carlsbad, CA, USA) and nucleophosmin/B23 was purchased from Abcam (Cambridge, UK). HKII antibody was purchased from Cell Signaling (Danvers, MA, USA).

\section{Immunohistochemistry}

Immunohistochemistry was performed using a Histofine streptavidin-biotin-peroxidase Kit (Nichirei, Tokyo, Japan) as previously described[18]. Briefly, 
4- $\mu \mathrm{m}$ thick sections, prepared from $10 \%$ formalin-fixed, paraffin embedded tissues, were deparaffinized in xylene and dehydrated through ethanol. Next, the sections were blocked, followed by incubation with anti-uMtCK antibody for $2 \mathrm{~h}$. Sections were then incubated with the secondary antibody for 20 min at RT and the reaction products were visualized by the chromogen 3,3'-diaminobenzidine tetrahydrochloride. All sections were counterstained with hematoxylin.

\section{Immunohistochemical analysis}

We evaluated uMtCK protein expression in normal prostatic epithelium and cancerous tissues derived from patients. For each case, we determined the Allred score, which is a semi-quantitative system that takes into consideration the proportion of positive cells, scored on a scale of $0-5$, and staining intensity, scored on a scale of $0-3$. The proportion and intensity were then summed to produce total scores of 0 or 2 through 8 . A score of 0 was regarded as negative; a score of 2 represented weak staining; scores of 3-6, moderate staining; and scores of 7-8, strong staining, as previously described[16]. Immunohistochemical scoring was performed by three independent researchers, including a pathologist.

\section{Cell culture, cell fractionation and immunob- lotting}

The human prostate cancer cell lines LNCaP, 22Rv1, PC3, and DU145 and the primary prostate epithelial cell line RWPE-1 were used in this study as previously described[18]. Cell fractionation and immunoblotting were performed as also previously described[18].

\section{Immunofluorescence}

Cells grown on glass coverslips were incubated for $15 \mathrm{~min}$ with a mitochondria-staining dye, MitoTracker Red (Invitrogen), fixed with $4 \%$ paraformaldehyde for $5 \mathrm{~min}$, and permeabilized in $0.01 \%$ Triton X-100-PBS ( $\mathrm{pH} \mathrm{7.4)} \mathrm{for} 10 \mathrm{~min}$ as previously described[18]. Coverslips were examined with a fluorescence microscope equipped with a Keyence inverted stand[18, 19].

\section{ATP quantification}

Cellular ATP was quantified using an ATP determination kit according to the manufacturer's instructions (Promega, San Luis Obispo, CA, USA). Briefly, cells, which had been plated at equal densities, were lysed in passive lysis buffer. Equal volumes of cell lysate were then added to the standard reaction solution, and luminescence was measured and normalized to the protein amount of each lysate.

\section{Statistical analysis}

The statistical analyses were performed using JMP version 7.0.1. The $\chi^{2}$ test was used to examine correlations between immunohistochemical uMtCK expression and clinicopathological parameters. Disease-free survival was defined as the period between surgery and the date of the last follow-up or PSA recurrence $(n=128)$. Survival curves were calculated by the Kaplan-Meier method, and compared by log-rank test. A two-sided $P$ value of $<0.05$ was considered statistically significant.

\section{Results}

\section{Expression of uMtCK protein is low in high Gleason grade prostate cancer tissue}

To explore the role for uMtCK in prostate cancer, we evaluated its expression profile in patient-derived prostate cancer tissues as well as matched normal tissues by immunohistochemistry. Figure 1 shows representative images for $\mathrm{uMtCK}$ protein expression in normal and cancerous prostate tissues. We found that $\mathrm{uMtCK}$ expression was mainly localized in the cytoplasm of epithelial cells (Fig. 1A, B). The luminal epithelial cells of normal prostate glands showed strong positivity. Similarly, in low Gleason grade cancer tissues (Gleason score: $\leq 3+4$ ), strong uMtCK expression was observed in the cytoplasm (Fig. 1C, D). In contrast, uMtCK expression in high Gleason grade prostate carcinoma tissues (Gleason score: $\geq$ $4+3$ ) was very weak (Fig. 1E, F). Because Gleason score is higher as prostate cancer advances, these results suggest that uMtCK expression is downregulated as prostate cancer progresses.

\section{Correlations between uMtCK expression and clinicopathological factors}

The correlations between uMtCK expression and clinicopathologic parameters are summarized in Table 1. Notably, we found that uMtCK expression was significantly decreased in prostate cancer tissues with higher Gleason scores, a measure of prostate cancer stage, compared with those with lower Gleason scores $(P=0.0014)$. uMtCK expression was also significantly decreased in poorly differentiated prostate cancer tissues compared with well-differentiated cancer tissues $(P=0.0017)$. No significant correlations were identified between uMtCK expression and patient age, pre-operative PSA concentration, surgical margin, or lymphovascular invasion. Thus, these data further support that uMtCK is downregulated as prostate cancer advances. 

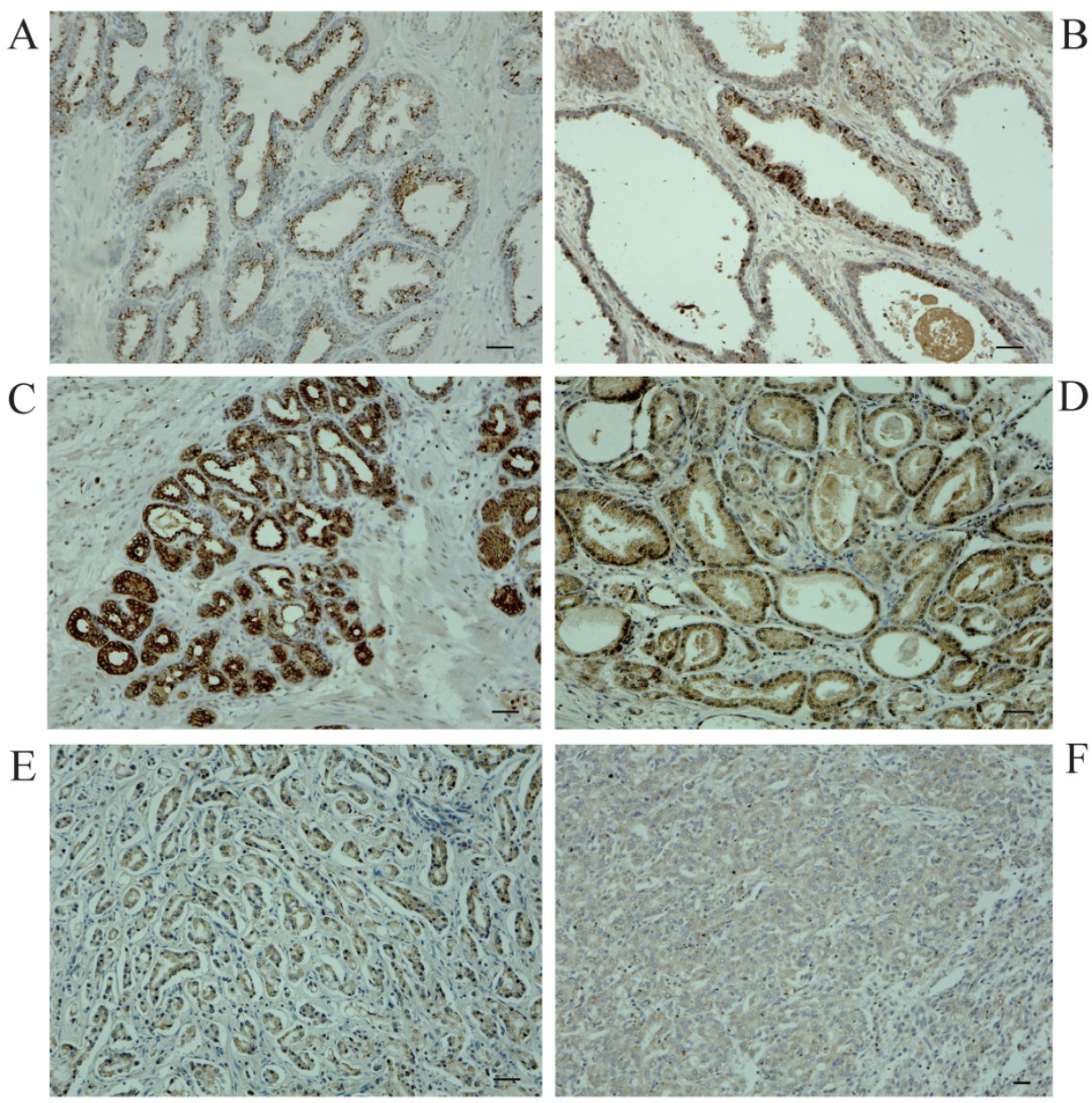

Figure 1. Immunohistochemical examination of uMtCK expression in patient-derived normal and cancerous prostate tissues. Immunohistochemistry was used to analyze uMtCK in (A, B) benign gland tissues, (C, D) low Gleason grade prostate cancer tissues, and (E, F) high Gleason grade prostate cancer tissues. Scale bar $=40 \mu \mathrm{m}$.

Table 1. Relationships between mtCK expression and clinicopathological characteristics

\begin{tabular}{|c|c|c|c|c|}
\hline \multirow[b]{2}{*}{ Variable } & \multicolumn{4}{|c|}{ mtCK expression } \\
\hline & $\mathrm{n}(\%)$ & Weak Mod & Strong & $P$-value \\
\hline \multicolumn{5}{|l|}{ Age } \\
\hline$<70$ & $99(66.9)$ & $75(50.7)$ & $24(16.2)$ & 0.76 \\
\hline$\geq 70$ & $49(33.1)$ & $36(24.3)$ & $13(8.8)$ & \\
\hline \multicolumn{5}{|l|}{ Gleason score } \\
\hline$\leq 3+4$ & $103(69.9)$ & $70(47.3)$ & $33(22.3)$ & $0.0014^{\dagger}$ \\
\hline$\geq 4+3$ & $45(30.1)$ & $41(27.7)$ & $4(2.7)$ & \\
\hline \multicolumn{5}{|c|}{ Pre-operative PSA (ng/ml) } \\
\hline$<10$ & $89(62.2)$ & $65(45.4)$ & $24(16.8)$ & 0.53 \\
\hline$\geq 10$ & $54(37.8)$ & $42(29.4)$ & $12(8.4)$ & \\
\hline \multicolumn{5}{|l|}{ Pathologic stage } \\
\hline pT2 & $101(68.2)$ & $76(51.4)$ & $25(16.9)$ & 0.91 \\
\hline $\mathrm{pT} 3 \sim \mathrm{T} 4$ & $47(31.8)$ & $35(23.7)$ & $12(8.1)$ & \\
\hline \multicolumn{5}{|l|}{ Differentiation } \\
\hline well & $101(68.6)$ & $68(46.4)$ & $33(22.1)$ & $0.0017 t^{\dagger}$ \\
\hline poorly & $46(31.4)$ & $42(28.5)$ & $4(2.9)$ & \\
\hline \multicolumn{5}{|c|}{ Lymphovasucular Invasion } \\
\hline negative & $136(91.9)$ & $102(68.9)$ & $34(22.9)$ & 1.0 \\
\hline positive & $12(8.1)$ & $9(6.8)$ & $3(2.0)$ & \\
\hline \multicolumn{5}{|l|}{ Relapse } \\
\hline$(-)$ & 115 (81.6) & $83(58.9)$ & $32(22.7)$ & 0.064 \\
\hline$(+)$ & $26(18.4)$ & $23(16.3)$ & $3(2.1)$ & \\
\hline
\end{tabular}

'Statistically significant $\left(\chi^{2}\right.$ test)

\section{Survival analysis of localized prostate carci- noma patients with high or low uMtCK ex- pression}

Because loss of uMtCK expression correlated with prostate cancer progression, we examined differences in survival between patients with high or low expression of uMtCK. Kaplan-Meier survival curve analyses of 128 patients with prostate cancer in which PSA recurrence-free survival rate was used as an end point showed that high uMtCK expression slightly, but not significantly, reduced the PSA recurrence-free survival rate compared with patients with weak to moderate uMtCK expression (log-rank test, $P=0.0852$ ) (Supplemental Fig. S1).

\section{Expression of uMtCK in prostate cancer cell lines}

To further explore the relationship between MtCK and prostate tumor progression, we investigated MtCK expression in four human prostate cancer cell lines and a non-cancerous cell line. High uMtCK expression was detected in the non-cancerous cell line 
RWPE-1 as well as in prostate cancer cell lines 22Rv1 and LNCaP. In contrast, uMtCK expression was comparatively lower in PC3 and DU145 cell lines (Fig. 2A). Similarly, the expression of sMtCK was detectable in 22Rv1 and LNCaP cells, but absent in PC3 and DU145 cells. However, HKII, a marker of glycolysis, was strongly expressed in PC3 and DU145 cells, but was lower in RWPE-1 cells (Fig. 2A). We divided the cells into two groups: the high uMtCK expressing cells (LNCaP and 22Rv1 cells) and the low uMtCK expressing cells (PC3 and DU145 cells). Moreover, because HKII is associated with glycolysis, these results suggest that there may be a switch in the energy production process used in later stages of prostate cancer.

The tumor suppressor $\mathrm{p} 53$ has been shown to be involved in the repression of cytosolic brain-type CK transcription. Thus, we next investigated whether its expression might be related to expression of $\mathrm{uMtCK}$ in prostate cancer cell lines. The expression of wild-type p53 was observed in normal RWPE-1 and uMtCK expressing cell lines, whereas the expression of mutant p53 was observed in the DU145 cell line (Fig. 2A). There was no expression of p53 in PC3 cells because of depletion of the p53 gene[20]. These results suggest that uMtCK expression in prostate cancer might be regulated by p53, and mutation or loss of p53 may lead to its decreased expression.

\section{Expression of mitochondrial OXPHOS pro- teins in prostate cancer cell lines}

Because MtCK activity is linked to mitochondrial ATP synthetase, we measured the expression of several members of the respiratory chain using western blot (Fig. 2B). Although NDUFA9 (complex I) expression was slightly downregulated in the PC3 and DU145 cell lines compared with other cell lines, there were no differences in the expression levels of the mtDNA-encoded proteins, COXI, COXIII, nuclear-encoded complex $\mathrm{V}$, and UQCRSF1, or in the level of the mitochondrial protein VDAC. These results suggest that the expression levels of OXPHOS proteins are not significantly correlated with uMtCK protein levels in prostate cancer cells.

\section{uMtCK is localized in mitochondria}

During tumor progression, some proteins change localization and function. To examine any potential changes in uMtCK, we investigated uMtCK localization in the prostate cancer cell lines. Immunocytochemistry showed that that uMtCK staining was granular in 22Rv1 and LNCaP cells, as well as in RWPE-1 cells (Fig. 3A). Moreover, it completely co-localized with the MitoTracker Red dye, suggesting that uMtCK is exclusively localized in mitochondria. HKII staining was granular in all four cell lines (Fig. 3B) and completely co-localized with MitoTracker Red dye, suggesting that HKII is also exclusively localized at mitochondria.

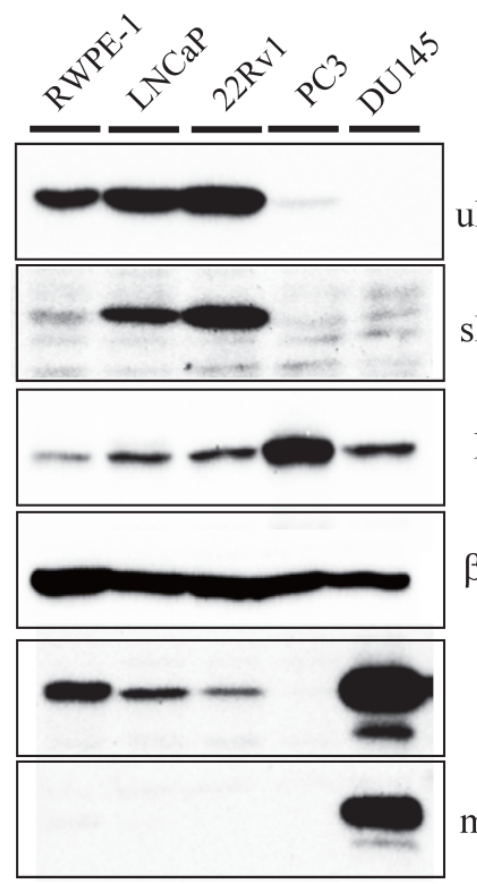

A

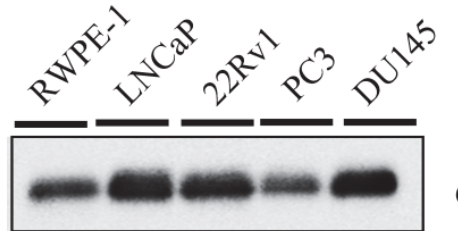

COX I (complex IV)

uMtCK

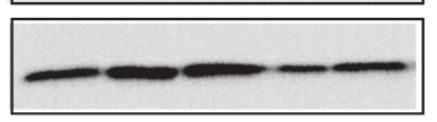

COX II (complex IV)

sMtCK

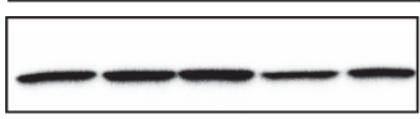

UQCRSF1(complex III)

HK II

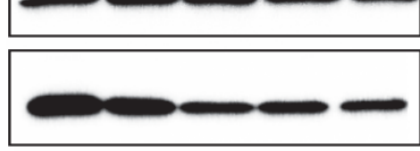

VDAC

$\beta$-actin

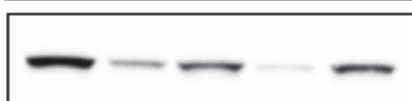

SDHA (complex II)

p53

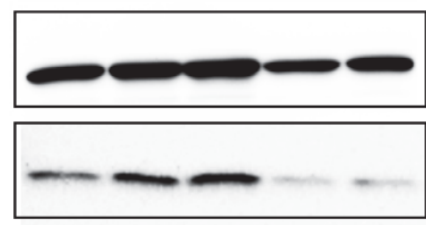

Complex V

mut p53

NDUFA9 (complex I)

Figure 2. Expression of uMtCK in prostate cancer cell lines. (A) Western blot analysis of uMtCK, sMtCK, HKII and p53 protein expression levels in prostate cancer and non-cancerous cell lines as indicated. $\beta$-actin was used as the internal control. (B) The protein expression levels of the indicated mitochondrial proteins were also measured in lysates prepared from the indicated prostate cancer cell lines. 
The intracellular localizations of $\mathrm{uMtCK}$ and HKII in LNCaP and PC3 cells were evaluated by cellular fractionation and western blotting. In both prostate cancer cell lines, uMtCK, along with p32, was found mostly in the mitochondrial fraction. HKII expression was also detected in the mitochondrial cell fractions of both cell lines, but was especially elevated in PC3 cells (Fig. 3C). These results suggest that $\mathrm{uMtCK}$ is localized at mitochondria in prostate cancer cell lines, as was HKII. HKII was predominantly overexpressed in the low uMtCK expressing PC3 cell line. Thus these data suggest that although the localization of proteins controlling OXPHOS and glycolytic ATP production are not altered in prostate cancer, glycolytic ATP production might be increased in low uMtCK expressing prostate cancer as indicated by increased HKII expression levels.

A
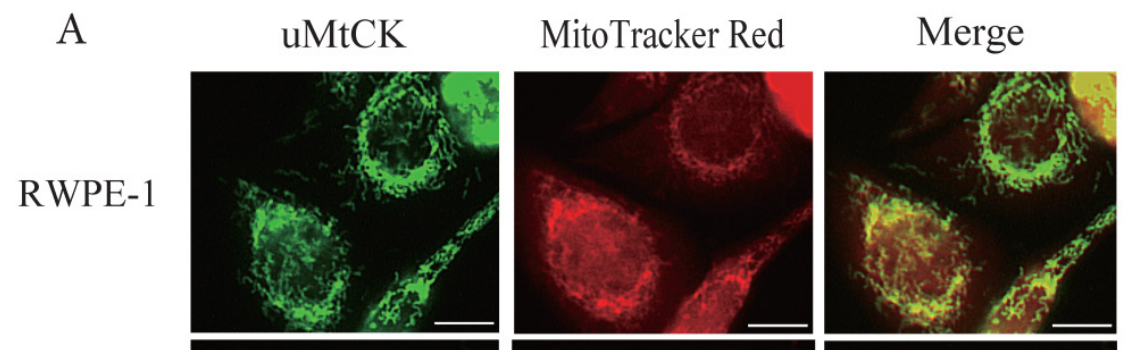

LNCaP
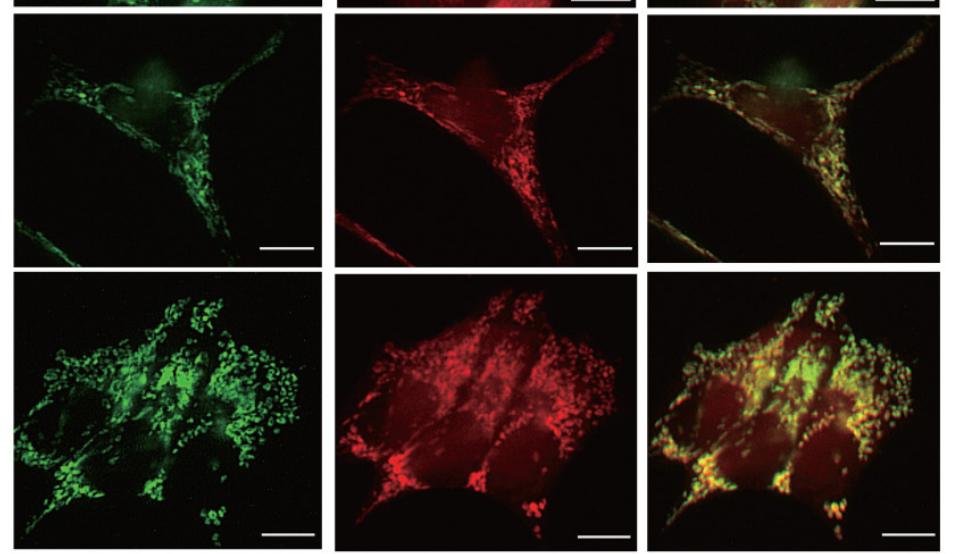

$22 \mathrm{Rv} 1$
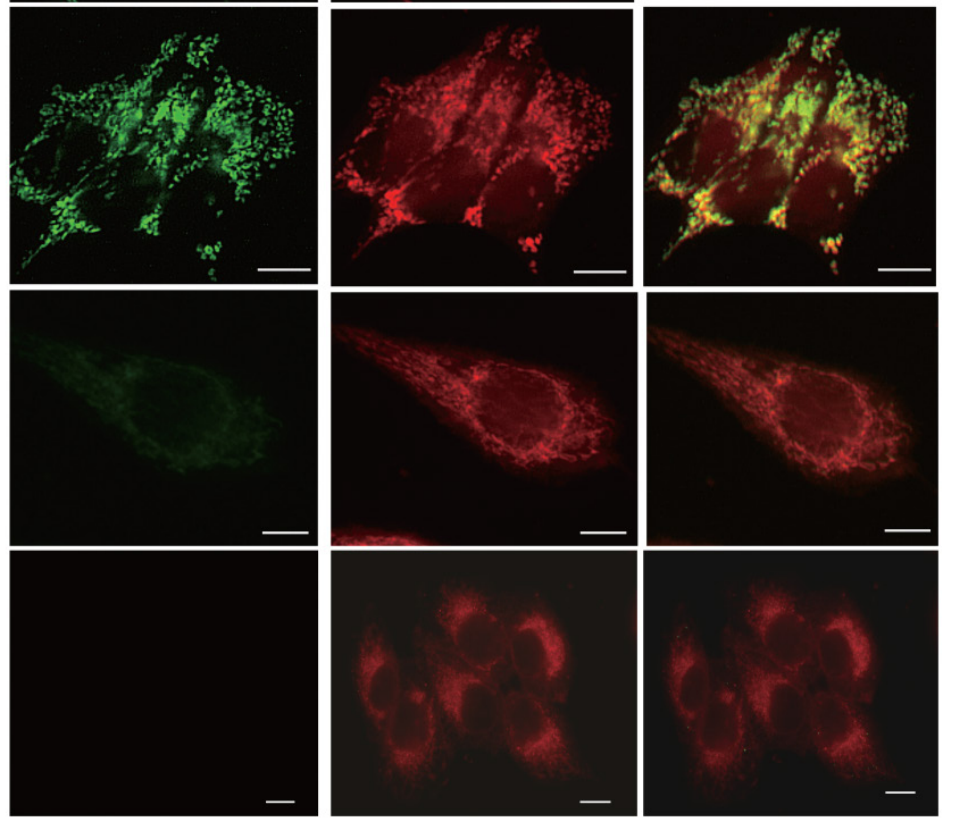


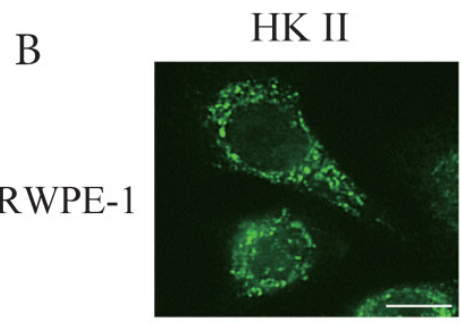

MitoTracker Red Merge
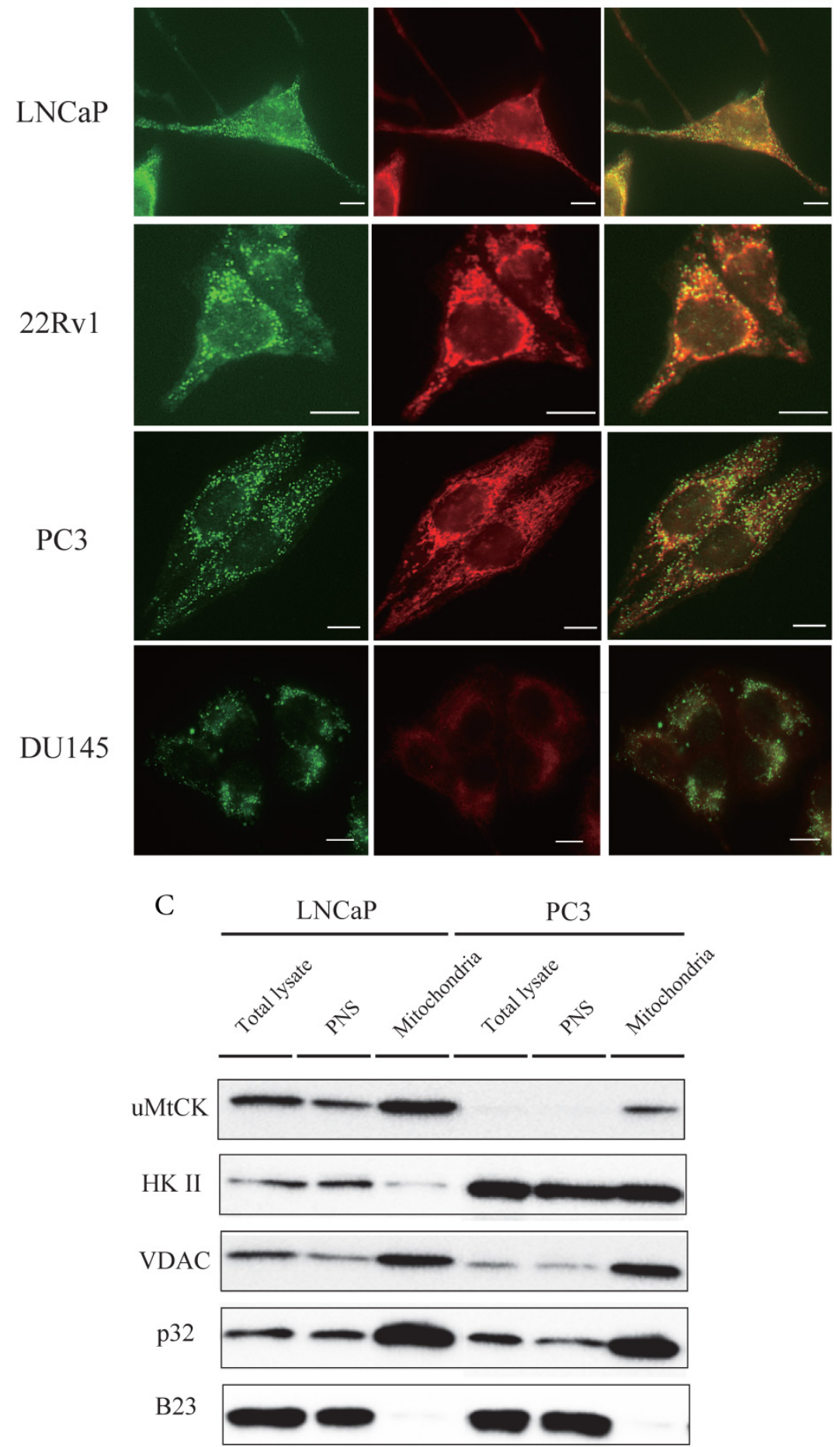

Figure 3. Immunocytochemistry of uMtCK and HKII in prostate cancer cells. (A) Mitochondria and uMtCK were visualized by immunocytochemistry using MitoTracker Red (middle panel) and uMtCK-specific antibody (left panel), respectively, in the indicated cell lines. (B) Mitochondria and HKII were visualized by immunocytochemistry using MitoTracker Red (middle panels) and HKII-specific antibody (left panel), respectively, in the indicated cell lines. Scale bar $=10 \mu \mathrm{m}$. (C) Subcellular localization of uMtCK was examined in prostate cancer cell lysates that had been separated into total, post nuclear supernatant (PNS), and mitochondrial fractions. The indicated proteins were detected by immunoblotting. The expression levels of $\mathrm{p} 32$ and VDAC were also detected as markers for mitochondria, whereas B23 (nucleophosmin) was used as a marker for nuclear and cytosolic localization. 

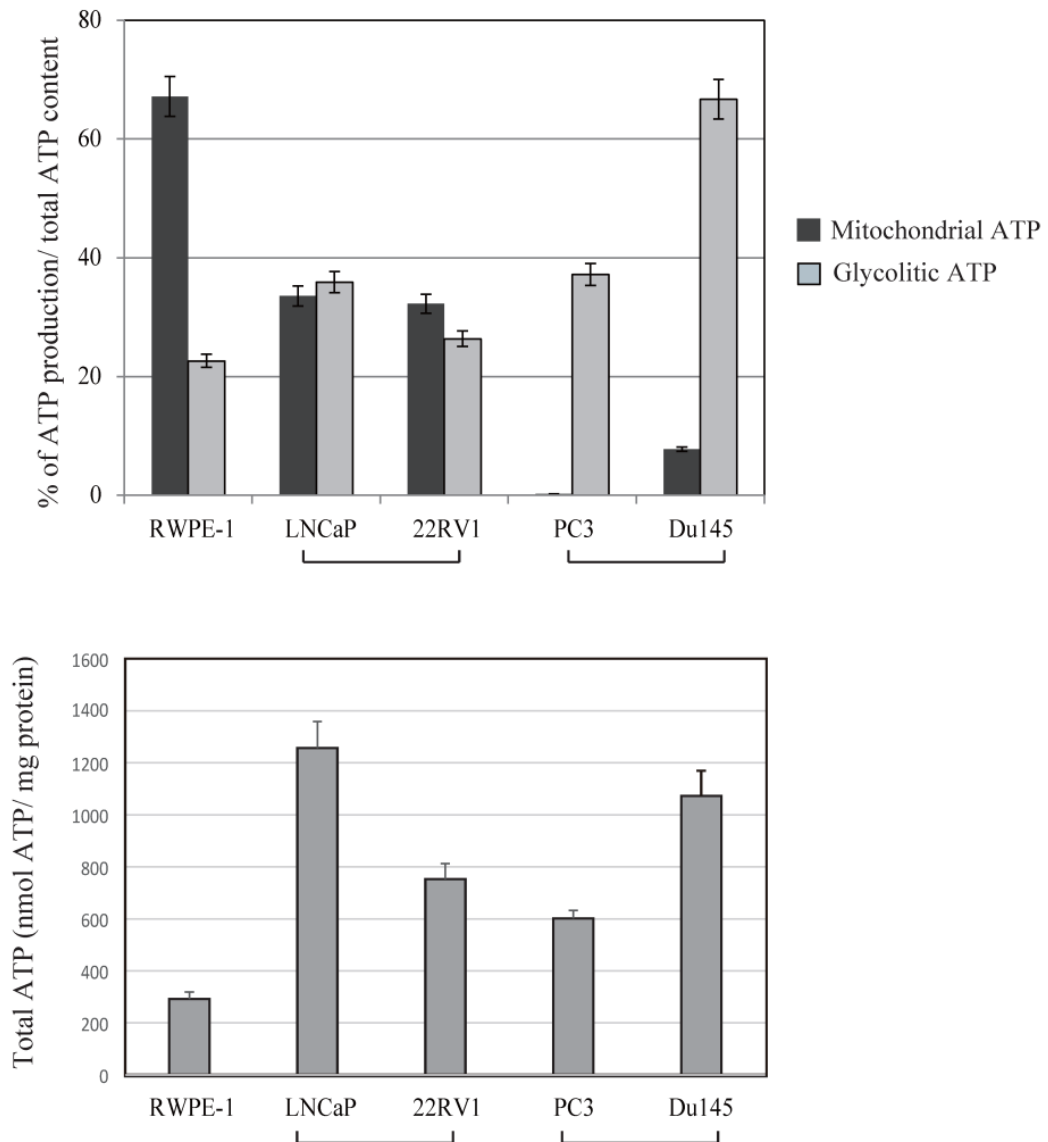

Figure 4. Effects of 2-deoxy-D-glucose (2DG) $(20 \mathrm{mM})$ and oligomycin $(10 \mu \mathrm{M})$ on intracellular ATP content in prostate cancer cell lines. The ATP content in untreated cells is presented in lane 1. The ATP concentration of untreated cells was subtracted from that of 2DG-treated cells for assessment of glycolytic ATP production. The ATP concentration in 2DG-treated cells was subtracted from that in cells treated with 2DG + oligomycin to determine mitochondrial ATP production. Lower panel showed total ATP production within each cell lines. The ATP response was measured using a Luminescence ATP assay kit. Data show the mean \pm SD of triplicate experiments.

\section{Discussion}

In this study, we investigated the status of $\mathrm{uMtCK}$ in prostate cancer during its malignant progression. We found that (i) the expression of uMtCK is significantly lower in patient-derived high Gleason grade carcinomas than in matched normal prostate tissues; (ii) high uMtCK expression might be regulated by 533 status; and (iii) in low uMtCK expressing prostate carcinoma cell lines, the glycolysis-associated protein HKII is overexpressed and localized in the mitochondrial outer membrane. Thus, for the first time, our study demonstrates relationships between uMtCK expression and both clinicopathological factors and survival in prostate cancer. Moreover, the decreased expression levels of uMtCK in high Gleason grade human prostate cancer suggest that it might be a potential molecular marker for poor prognosis.

Onda and colleagues previously reported that uMtCK is downregulated in oral squamous cell carcinoma in correlation with tumor differentiation $(P<0.0001)$. The authors proposed that the downregulation of uMtCK might be due to the frequent inac- tivation of the uMtCK gene during oral carcinogenesis, which occurs via an epigenetic mechanism and leaks to blockade of apoptosis[8]. It was reported that MtCK expression were increased in human hepatocellular carcinoma and mouse fibrotic liver [21, 22]. It might be speculated that MtCK expression were regulated dependency on tissue specificity or stage of tumor. Another regulator of MtCK expression is the p53 tumor suppressor, which acts as a transcriptional activator of the uMtCK gene. Various studies have shown that the level of p53 protein is decreased owing to mutation of its gene in many tumor cells[23, 24]. Hence, it can be assumed that deregulation of p53 protein may underscore the lower level of uMtCK production observed in differentiated prostate cancer both in vitro and in clinical samples in our study.

In mammalian cells, there are two main ways to generate energy in the form of ATP from glucose: OXPHOS and glycolysis. OXPHOS occurs in mitochondria with carbon dioxide and water as end products, whereas glycolysis converts glucose to lactic acid and takes place in the cytoplasm. In tumorigenesis, glucose is metabolized into lactic acid instead of carbon dioxide and water even in the presence of ox- 
ygen. This phenomenon is known as the Warburg effect[5, 25-27].

Our results showed that uMtCK protein expression was downregulated in prostate tumorigenesis, whereas HKII expression was increased. HKII catalyzes the first step of glycolysis, i.e. the phosphorylation of glucose to G-6-P. In many cancers, including colon, prostate, lymphoma, glioma, gastric adenomas, carcinomas and breast cancers, the propensity of malignant cells for high glycolytic activity is dependent on the marked overexpression of HKII [13, 28, 29]. The binding of HKII to mitochondria has been shown to inhibit Bax-induced cytochrome $\mathrm{c}$ release and thereby impair apoptosis [30]. A previous study demonstrated that tubulin and VDAC form a supercomplex with $\mathrm{MtCK}$, which is functionally and structurally coupled to the ATP synthasome [31]. In HL-1 cells, this VDAC-HKII complex is significantly modified. Tubulin is replaced by HK and MtCK is lacking, resulting in direct use of mitochondrial ATP for glycolytic lactate production, thereby contributing to the Warburg effect [31]. These observations indicate that high Gleason grade prostate carcinoma, such as that represented by PC3 cells, demonstrate an ATP demand exchange (Fig. 5).
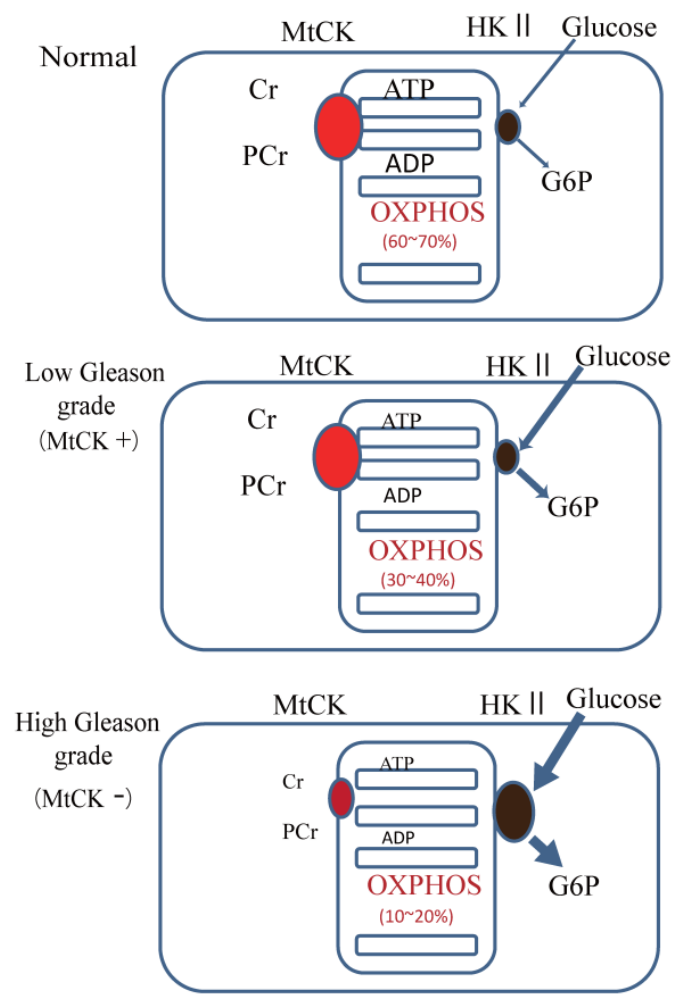

Figure 5. Model of the expression of uMtCK in prostate cancer progression. Top panel: in normal cells, uMtCK is expressed in the intermembrane space of mitochondria where it transfers the phosphate group of ATP to creatine to yield phosphocreatine. This, in turn, is used as an ATP stock. Middle panel: similarly, in low Gleason grade carcinoma, uMtCK is expressed in mitochondria and functions in energy transfer. Bottom panel: in high Gleason grade carcinoma, uMtCK is not expressed. Instead, HKII is localized at mitochondria, suggesting a shift to increased aerobic glycolysis.
In summary, our findings show that whereas uMtCK expression is readily detectable in normal prostate and low Gleason score prostate cancer, it progressively decreases as prostate cancer progresses until it is virtually absent in high Gleason grade prostate carcinoma. These findings suggest that uMtCK may be a novel marker of clinical progression in prostate cancer. Moreover, its unique localization in tumors may make uMtCK a useful target in the diagnosis and therapy selection for patients with prostate tumors and possibly for other tumors.

\section{Supplementary Material}

Table S1 and Fig.S1.

http://www.jcancer.org/v07p0050s1.pdf

\section{Acknowledgements}

The authors would like to acknowledge the technical expertise of the Support Center for Education and Research, Kyushu University.

\section{Financial support}

This work was supported in part by Grants-in-Aid for Scientific Research from the Ministry of Education, Culture, Sports, Science and Technology of Japan (grant numbers \#25253041 and \#24590387).

\section{Competing Interests}

The authors have declared that no competing interest exists.

\section{References}

1. Jemal A, Siegel R, Ward E, Murray T, Xu J, Smigal C, et al. Cancer statistics, 2006. CA Cancer J Clin. 2006; 56: 106-30.

2. Giovannucci E, Liu Y, Platz EA, Stampfer MJ, Willett WC. Risk factors for prostate cancer incidence and progression in the health professionals follow-up study. Int J Cancer. 2007; 121: 1571-8.

3. Hebert JR, Hurley TG, Olendzki BC, Teas J, Ma Y, Hampl JS. Nutritional and socioeconomic factors in relation to prostate cancer mortality: a cross-national study. J Natl Cancer Inst. 1998; 90: 1637-47.

4. Chen JZ, Kadlubar FF. Mitochondrial mutagenesis and oxidative stress in human prostate cancer. J Environ Sci Health C Environ Carcinog Ecotoxicol Rev. 2004; 22: 1-12.

5. Warburg O. On the origin of cancer cells. Science. 1956; 123: 309-14.

6. Wallimann T, Wyss M, Brdiczka D, Nicolay K, Eppenberger HM. Intracellular compartmentation, structure and function of creatine kinase isoenzymes in tissues with high and fluctuating energy demands: the 'phosphocreatine circuit' for cellular energy homeostasis. Biochem J. 1992; 281 (Pt 1): 21-40.

7. Haas RC, Korenfeld C, Zhang ZF, Perryman B, Roman D, Strauss AW. Isolation and characterization of the gene and cDNA encoding human mitochondrial creatine kinase. J Biol Chem. 1989; 264: 2890-7.

8. Onda T, Uzawa K, Endo Y, Bukawa H, Yokoe H, Shibahara T, et al. Ubiquitous mitochondrial creatine kinase downregulated in oral squamous cell carcinoma. Br J Cancer. 2006; 94: 698-709.

9. Schlattner U, Tokarska-Schlattner M, Wallimann T. Mitochondrial creatine kinase in human health and disease. Biochim Biophys Acta. 2006; 1762: 164-80.

10. Kekelidze T, Khait I, Togliatti A, Benzecry JM, Wieringa B, Holtzman D. Altered brain phosphocreatine and ATP regulation when mitochondrial creatine kinase is absent. J Neurosci Res. 2001; 66: 866-72.

11. Kanemitsu F, Kawanishi I, Mizushima J, Okigaki T. Mitochondrial creatine kinase as a tumor-associated marker. Clin Chim Acta. 1984; 138: 175-83.

12. Pratt R, Vallis LM, Lim CW, Chisnall WN. Mitochondrial creatine kinase in cancer patients. Pathology. 1987; 19: 162-5.

13. Mathupala SP, Ko YH, Pedersen PL. Hexokinase II: cancer's double-edged sword acting as both facilitator and gatekeeper of malignancy when bound to mitochondria. Oncogene. 2006; 25: 4777-86. 
14. Nakashima RA, Mangan PS, Colombini M, Pedersen PL. Hexokinase receptor complex in hepatoma mitochondria: evidence from $\mathrm{N}, \mathrm{N}$-dicyclohexylcarbodiimide-labeling studies for the involvement of the pore-forming protein VDAC. Biochemistry. 1986; 25: 1015-21.

15. Epstein JI, Allsbrook WC, Jr., Amin MB, Egevad LL. The 2005 International Society of Urological Pathology (ISUP) Consensus Conference on Gleason Grading of Prostatic Carcinoma. Am J Surg Pathol. 2005; 29: 1228-42.

16. Song Y, Oda Y, Hori M, Kuroiwa K, Ono M, Hosoi F, et al. N-myc downstream regulated gene-1/Cap43 may play an important role in malignant progression of prostate cancer, in its close association with E-cadherin. Hum Pathol. 2010; 41: 214-22.

17. Kanki T, Ohgaki K, Gaspari M, Gustafsson CM, Fukuoh A, Sasaki N, et al. Architectural role of mitochondrial transcription factor A in maintenance of human mitochondrial DNA. Mol Cell Biol. 2004; 24: 9823-34.

18. Amamoto R, Yagi $M$, Song $Y$, Oda $Y$, Tsuneyoshi $M$, Naito $S$, et al. Mitochondrial p32/C1QBP is highly expressed in prostate cancer and is associated with shorter prostate-specific antigen relapse time after radical prostatectomy. Cancer Sci. 2011; 102: 639-47.

19. Uchiumi T, Ohgaki K, Yagi M, Aoki Y, Sakai A, Matsumoto S, et al. ERAL1 is associated with mitochondrial ribosome and elimination of ERAL1 leads to mitochondrial dysfunction and growth retardation. Nucleic Acids Res. 2010; 38: 5554-68.

20. Arah IN, Song K, Seth P, Cowan KH, Sinha BK. Role of wild-type p53 in the enhancement of camptothecin cytotoxicity against human prostate tumor cells. Anticancer Res. 1998; 18: 1845-9.

21. Uranbileg B, Enooku K, Soroida Y, Ohkawa R, Kudo Y, Nakagawa H, et al. High ubiquitous mitochondrial creatine kinase expression in hepatocellular carcinoma denotes a poor prognosis with highly malignant potential. Int J Cancer. 2014; 134: 2189-98.

22. Meffert G, Gellerich FN, Margreiter R, Wyss M. Elevated creatine kinase activity in primary hepatocellular carcinoma. BMC Gastroenterol. 2005; 5: 9.

23. Zhao J, Schmieg FI, Logsdon N, Freedman D, Simmons DT, Molloy GR. p53 binds to a novel recognition sequence in the proximal promoter of the rat muscle creatine kinase gene and activates its transcription. Oncogene. 1996; 13: 293-302.

24. Tamir Y, Bengal E. p53 protein is activated during muscle differentiation and participates with MyoD in the transcription of muscle creatine kinase gene. Oncogene. 1998; 17: 347-56.

25. Stern R, Shuster S, Neudecker BA, Formby B. Lactate stimulates fibroblast expression of hyaluronan and CD44: the Warburg effect revisited. Exp Cell Res. 2002; 276: 24-31.

26. Semenza GL. Tumor metabolism: cancer cells give and take lactate. J Clin Invest. 2008; 118: 3835-7.

27. Herrmann PC, Herrmann EC. Oxygen metabolism and a potential role for cytochrome c oxidase in the Warburg effect. J Bioenerg Biomembr. 2007; 39: 247-50.

28. Bryson JM, Coy PE, Gottlob K, Hay N, Robey RB. Increased hexokinase activity, of either ectopic or endogenous origin, protects renal epithelial cells against acute oxidant-induced cell death. J Biol Chem. 2002; 277: 11392-400.

29. Gottlob K, Majewski N, Kennedy S, Kandel E, Robey RB, Hay N. Inhibition of early apoptotic events by Akt/PKB is dependent on the first committed step of glycolysis and mitochondrial hexokinase. Genes Dev. 2001; 15: 1406-18.

30. Pastorino JG, Shulga N, Hoek JB. Mitochondrial binding of hexokinase II inhibits Bax-induced cytochrome c release and apoptosis. J Biol Chem. 2002; 277: 7610-8.

31. Saks V, Guzun R, Timohhina N, Tepp K, Varikmaa M, Monge C, et al. Structure-function relationships in feedback regulation of energy fluxes in vivo in health and disease: mitochondrial interactosome. Biochim Biophys Acta. 2010; 1797: 678-97. 